\title{
The Impact of Aflibercept on Diabetic Macular Edema Patients Refractory to Ranibizumab in a Tertiary Care Eye Specialist Hospital, Saudi Arabia
}

\author{
Abdullah AlHumaidan*, Pharmacy \\ Department, King Khaled Eye Specialist \\ Hospital, Riyadh, SAUDI ARABIA. \\ Sitah AIZuman, Pharmacy Department, \\ King Khaled Eye Specialist Hospital, Riyadh, \\ SAUDI ARABIA. \\ Muhammad Haris Mazhar, Pharmacy \\ Department, King Khaled Eye Specialist \\ Hospital, Riyadh, SAUDI ARABIA. \\ Arwa A AIDahash, Pharmacy \\ Department, King Khaled Eye Specialist \\ Hospital, Riyadh, SAUDI ARABIA. \\ Wejdan AlMussalam, Pharmacy \\ Department, King Khaled Eye Specialist \\ Hospital, Riyadh, SAUDI ARABIA. \\ Hassan AIDhibi, Vitreo-retina Department, \\ King Khaled Eye Specialist Hospital, Riyadh, \\ SAUDI ARABIA \\ Marco Mura, Vitreo-retina Department, \\ King Khaled Eye Specialist Hospital, Riyadh, \\ SAUDI ARABIA. \\ Loujain AlAbbasi, Diagnostic Imaging \\ Department, King Khaled Eye Specialist \\ Hospital, Riyadh, SAUDI ARABIA. \\ Rajiv Khandekar, Research Department, \\ King Khaled Eye Specialist Hospital, Riyadh, \\ SAUDI ARABIA.
}

\section{Correspondence:}

Dr. Abdullah AlHumaidan, PharmD,

Director of Pharmacy Department,

King Khaled Eye Specialist Hospital, Riyadh, SAUDI ARABIA.

Phone no: +966504417712

E-mail:ahumaidan@kkesh.med.sa

Received: 17-06-2021;

Accepted: 16-09-2021.

Copyright: ๑ the author(s),publisher and licensee Pharmacology, Toxicology and Biomedical Reports. This is an open-access article distributed under the terms of the Creative Commons Attribution NonCommercial License, which permits unrestricted non-commercial use, distribution, and reproduction in any medium, provided the original work is properly cited.

This is an open access article distributed under the terms of the Creative Commons AttributionNonCommercial-ShareAlike 4.0 License

\begin{tabular}{|l|l|}
\hline \multicolumn{2}{|c|}{ Access this article online } \\
\hline & www.ptbreports.org \\
\hline
\end{tabular}

\begin{abstract}
Aim: The purpose of this study is to see if switching from intravitreal Ranibizumab, an anti-Vascular Endothelial Growth Factor (anti-VEGF), to Aflibercept is evidence-based in the diabetic Saudi population. We compared the efficacy of three injections of the Aflibercept treatment regimen in diabetic macular edema (DME) cases refractory to Ranibizumab. Methods: In 2018, a retrospective single-arm cohort study was carried out. Cases of refractory DME were evaluated before and after three injections of Ranibizumab, as well as three Aflibercept treatment regimens. Optical Coherence Tomography (OCT) was used to investigate changes in Central Macular Thickness (CMT) (anatomic outcome). In addition, changes in the CMT and Best Corrected Visual Acuity (BCVA) (functional outcome) were assessed. Results: The cohort consisted of 74 eyes from 74 DME patients. As an anatomical indicator, the mean CMT decreased from $482 \pm 153 \mu$ to $385.6 \pm 134.6 \mu$ after three injections of Ranibizumab. In contrast, with Aflibercept treatment, the mean CMT decreased to $370.2 \pm 116.9 \mu$. The decrease in CMT was statistically significant [94.6 $(95 \%$ confidence interval (CI): 54.8; 134.3] (P 0.001). In addition, BCVA improved by two or more lines in $17(23 \%)$ of the eyes treated with Aflibercept, remained unchanged in $40(54 \%)$ of the eyes, and decreased in $17(23 \%)$ of the eyes. Conclusion: The anatomic successes of both anti-VEGF (Ranibizumab and Aflibercept) treatment regimens in Saudi patients with DME are promising. The Aflibercept treatment regimen improves success in refractory DME cases, but the visual improvement appears to be limited.

Key words: Aflibercept, Ranibizumab, Diabetic Macular Edema (DME), Anti Vascular Endothelial Growth Factors (Anti-VEGF), Diabetic retinopathy, Diabetes.
\end{abstract}

\section{INTRODUCTION}

Diabetic Macular Edema (DME) impairs central vision due to disruption of the blood-retinal barrier, resulting in fluid accumulation within the retinal layers at the macula. ${ }^{1,2}$ Anti-Vascular Endothelial Growth Factors (VEGF) such as Bevacizumab, Ranibizumab, and Aflibercept are more effective than laser in improving vision in DME patients. ${ }^{3,4}$ VEGF is a key mediator of abnormal vascular permeability in DME. ${ }^{5}$

Despite some documented limitations, the high cost of anti-VEGF treatments prompted the widespread use of Bevacizumab injection to treat DME. ${ }^{6}$ Several experts and ophthalmic societies have recommended that intravitreal anti-VEGF compounds be switched as needed..$^{7-9}$ However, factors such as glycemic control, oxidative stress, hypertension control, and lipid levels all impact the response to DR and DME treatment. ${ }^{10}$ As a result, healthcare providers will be interested in a DME treatment protocol for selecting anti-VEGF medications for treating refractory DME in the diabetic Saudi population.

Previous research has found that intravitreal Aflibercept improves vision and reduces macular edema in patients who received anti-VEGF therapy. ${ }^{11,12}$ To the best of our knowledge, no study has been conducted in the Saudi population on the effect of Aflibercept on DME in patients who were refractory to Ranibizumab treatment.

\section{METHODS}

In August 2018, our institute's ethical committee approved this retrospective study ( $\mathrm{R}-1846)$. A Waiver of Informed Consent was obtained due to the nature of the study. The data was gathered through a chart review. For analysis, personally identifiable information was delinked from the data. Patients were eligible if they were 18 years or older and had received at least three doses of Ranibizumab (Lucentis ${ }^{\oplus}$, Novartis Pharma Stein AG, Stein Switzerland) intravitreal injection$0.5 \mathrm{mg} / 0.05 \mathrm{~mL}$. They were followed by at least three doses of intravitreal Aflibercept (Eylea; Regeneron Pharmaceuticals Inc., New York, United States). The decision was made based on an assessment of disease progression after approximately 28 days of Ranibizumab injection. Patients with DME with CMT > 300um and/ or BCVA 20/25-20/200 Snellen equivalent monitored at our institution from February 2017 to January 2018 were also eligible. ${ }^{13}$ Patients were excluded if they had received fewer than three injections of Ranibizumab or Aflibercept. The pregnant had ocular comorbidities such as Uveitis or Retinal Vascular Occlusion, and DME with epiretinal membrane or vitreomacular traction, or had hazy media. Furthermore, patients were excluded if there was inconclusive information on best-corrected visual acuity (BCVA) or central macular thickness (CMT) before switching antiVEGF treatments or at the end of the study. The 
study's objective is to look at the short-term functional and anatomical outcomes of diabetic macular edema (DME) patients switched from Ranibizumab to Aflibercept.

Before and after Aflibercept treatment, the CMT was assumed to be $431.2 \pm 147.8$ and $321.4 \pm 98 \mu \mathrm{m}$, respectively. ${ }^{14}$ A one-armed cohort study requiring at least 58 eyes for evaluation before and after Aflibercept treatment was required to achieve a 95 percent confidence interval (CI) and 90 percent power. In addition, to compensate for data loss, the sample size was increased by $20 \%$. As a result, the final sample included at least 70 eyes.

The study's investigators included two retinal subspecialists, pharmacists and epidemiologists. Al-Rasheed et al. previously described our institution's intravitreal injection protocol. ${ }^{15}$ Our Aflibercept vial contains $4 \mathrm{mg} / 0.1 \mathrm{ml}$, and the recommended dosage per eye is $2 \mathrm{mg} / 0.05 \mathrm{ml}$. The intravitreal injection is performed as an outpatient procedure, and the intraocular pressure (IOP) and vision are monitored immediately following the process. Three intravitreal injections were given at onemonth intervals. The evaluation of the CMT and BCVA was scheduled one month after the completion of the entire course of three injections. The demographic information included age, gender, and the DMEaffected eye to which the anti-VEGF injection was administered, which could be either the right or left side.

At a 6-meter distance, BCVA was measured using a projection chart. The measurements were in feet. The BCVA was documented three times: at presentation, during the switch from Ranibizumab to Aflibercept, and one month after the third Aflibercept injection. Success was defined as improving more than two lines in BCVA at the last follow-up compared to BCVA before beginning Aflibercept treatment.

Optical coherence tomography (OCT) was used to assess CMT (Spectralis; Heidelberg Engineering, Heidelberg, Germany). The anatomical impact of Aflibercept was evaluated by comparing CMT when the intravitreal regimen was switched to CMT at the end of the study. Anatomical success was defined as a decrease in CMT, while failure was defined as either no change or CMT increase.

The data were gathered from the electronic case records of patients with DME treated during the study period. The information from a pretested data collection form was transferred using an Excel $^{\circ}$ spreadsheet (Microsoft Corp., Redmond, WA, USA). The information on intravitreal injection was also confirmed using the procedure room's operation logbook. The data was transferred to the Statistical Package for Social Studies after checking for consistency, duplication, and completion
(SPSS 26; IBM Corp., Armonk, NY, USA). The frequency and percentage proportion of qualitative data are presented. In addition, the mean and standard deviation of normally distributed quantitative data are shown. Matched pair analysis was used to examine the statistical validity of the outcome variables. The median and interquartile range (IQR) were calculated for non-normal quantitative data distributions. In order to compare outcome variables in subgroups, Mann-Whitney $P$ values were calculated using a non-parametric method. A $P$ value of 0.05 or less was considered statistically significant.

\section{RESULTS}

This study included 74 diabetic patients with DME, with a mean age of $68.2 \pm 8.1$ years. There were $51(69 \%)$ males, and $33(44.6 \%)$ of the total were right eye.

The median time between ophthalmic assessment for DME and the first Aflibercept injection was 23 days [interquartile range (IQR) 3.5; 35]. The median time between the first and second Aflibercept injections was 29 days (IQR 28; 35). The median time between the second and third Aflibercept injections was 28 days (IQR 28; 29). The median time between the third Aflibercept injection and the final assessment was 40 days (IQR 30; 58).

Table 1 compares CMT and grades of visual impairment (VI) based on the BCVA at presentation, at the time of the switch between anti-VEGF compounds, and at last follow up. After 3 Aflibercept injections, there was a statistically significant reduction in CMT compared to the baseline mean CMT. $(P<0.001)$.

\section{Anatomical impact of Aflibercept treatment regimen on DME refractory} to Ranibizumab treatment regimen:

After completing the Ranibizumab treatment regimen, 32 (43.2\%) eyes with DME had an increase in CMT, 37 (50\%) had a decrease in CMT, and data were not available for five eyes. After switching to and completing the Aflibercept treatment regimen, 58 (78.5\%) had a reduction in CMT compared to before the switch, 11 (14.9\%) had an increase in CMT, and data were not available for five eyes.

Only 3 (9.4\%) of the 32 eyes with increased CMT after Ranibizumab treatment had increased CMT after three injections of Aflibercept. Thus, 3 Aflibercept injections resulted in success by lowering CMT in 29 eyes. The status of CMT at presentation, at the switch, and after completion of anti-VEGF treatment is compared in Figure 1.

Table 1: Central macular thickness and visual impairment grades at presentation, at the time of switching from Ranibizumab to Aflibercept, and after three intravitreal injections of Aflibercept.

\begin{tabular}{|c|c|c|c|c|c|c|c|}
\hline $\begin{array}{l}\text { Central Macula Thickness } \\
\qquad(\mu)\end{array}$ & \multicolumn{2}{|c|}{ At presentation } & \multicolumn{2}{|c|}{$\begin{array}{l}\text { At the time of the switch of anti } \\
\text { VGEF }\end{array}$} & \multicolumn{2}{|c|}{$\begin{array}{c}\text { After three injections of } \\
\text { Aflibercept }\end{array}$} & Validation \\
\hline Mean & \multicolumn{2}{|c|}{481.7} & \multicolumn{2}{|c|}{469.7} & \multicolumn{2}{|c|}{382.5} & $P<0.001$ \\
\hline \multirow[t]{2}{*}{ Validation (matched pair) } & \multicolumn{6}{|c|}{ Difference of mean at presentation and after three injections of Aflibercept $=94.6(95 \%$ CI $54.8 ; 134.3) P<0.001$} & \\
\hline & \multicolumn{6}{|c|}{ Difference of mean at switch and after 3 injections of Aflibercept $=104.5$ (95\% CI 72.5 ; 136.4) $P<0.001$} & \\
\hline $20 / 20$ to $20 / 60$ & 21 & 28.4 & 22 & 29.7 & 30 & 40.5 & \multirow{4}{*}{$\begin{array}{c}\chi^{2}=7.3 \\
\mathrm{DF}=6 \\
P=0.3\end{array}$} \\
\hline$<20 / 60$ to $20 / 200$ & 44 & 59.5 & 38 & 51.4 & 30 & 40.5 & \\
\hline$<20 / 200$ to $20 / 400$ & 6 & 8.1 & 10 & 13.5 & 12 & 16.2 & \\
\hline$<20 / 400$ & 3 & 4.1 & 4 & 5.4 & 2 & 2.7 & \\
\hline
\end{tabular}

CI denotes confidence interval; $\mathrm{P}<0.05$ is statistically significan 


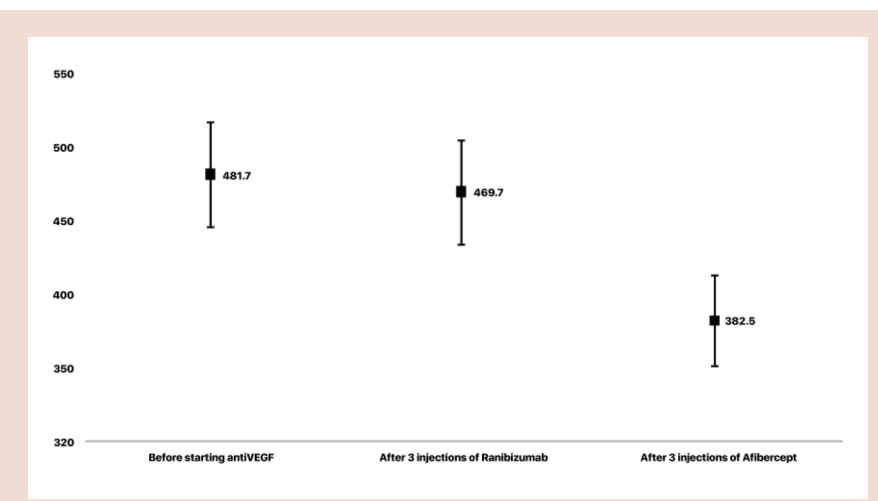

Figure 1: Central macula thickness (CMT) as measured by Optical Coherence Tomography in Eyes with diabetic macular edema before and after anti-vascular growth factor treatment regimen.

$\mathrm{X}$-axis shows different times when CMT was measured.

Y-axis shows CMT $(\mu \mathrm{m})$.

The lower and upper ends of the line depict the Interquartile range.

The central square symbol indicates the median value of CMT.

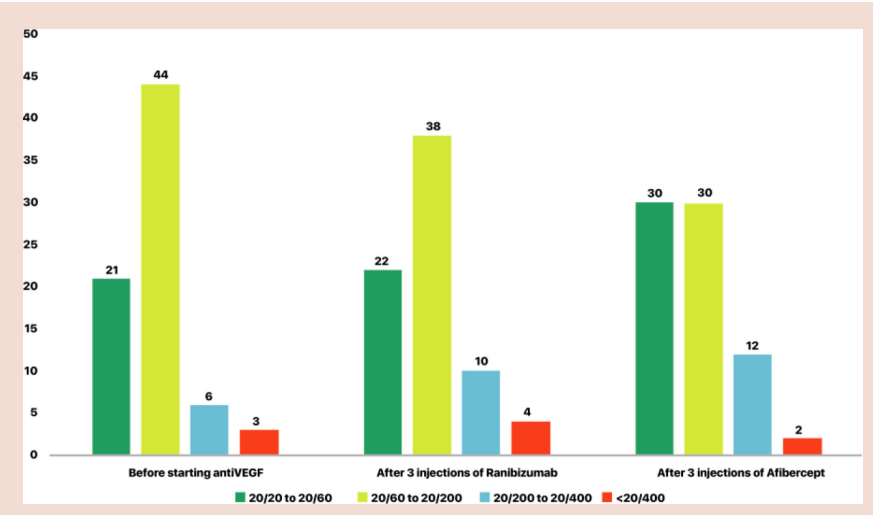

Figure 2: The Best-corrected distance visual acuity in eyes with diabetic macular edema before and after anti-vascular growth factor treatment regimen.

$\mathrm{X}$-axis shows the different times when central macula thickness was measured.

$\mathrm{Y}$-axis shows the number of eyes with different grades of visual impairment.

\section{Functional impact of Aflibercept treatment regimen on} DME refractory to Ranibizumab treatment regimen

BCVA improved by two or more lines in $17(23 \%)$ eyes treated with the Aflibercept regimen. However, BCVA remained unchanged in 40 (54\%) of the eyes after Aflibercept treatment and decreased in 17 (23\%) of the eyes.

Figure 2 shows a comparison of BCVA before and after anti-VEGF treatment. The difference in BCVA between the two anti-VEGF treatments for DME was not statistically significant. $(P=0.3)$

\section{Factors influencing the anatomical impact of Aflibercept treatment regimen}

Gender: In 49 male diabetic patients, the median decrease in CMT was $65.0 \mu \mathrm{m}$. (IQR 10.0; 165.5). In 20 female diabetic patients, the median decrease in CMT was $77.5 \mu \mathrm{m}$. (IQR 32.8; 229.7) (Mann Whitney $P=0.4$ )

\section{CMT before switching to Aflibercept}

There was a significant reduction in the CMT after three intravitreal injections of Aflibercept compared to CMT before starting Aflibercept treatment. (Wilcoxon signed-rank test $P$ 0.001).

Age of diabetic patient: The decline in CMT following the Aflibercept treatment regimen was not significantly related to the patient's age. (Pearson $P=0.85$ ).

\section{DISCUSSION}

The findings of this study show the utility of switching from Ranibizumab to Aflibercept treatment in patients with refractory DME. Although there was a significant anatomic success, visual gains were only moderate. CMT at the start of Aflibercept treatment was a determinant of success. To our knowledge, this is the first study in a Saudi population that evaluates the role of Aflibercept treatment in eyes with DME that is refractory to Ranibizumab treatment. The current study enrolled Saudi diabetic patients, who are known to have poor primary diabetes prevention measures. ${ }^{16}$

In our study, more than three-quarters of DME cases had a decrease in CMT at their last postoperative visit. Chen et al. reported success in lowering CMT in two-thirds of DME eyes. ${ }^{17}$ In Chen et al.'s study, $20 \%$ of the eyes were treated with Bevacizumab, while the rest were treated with Ranibizumab before switching to Aflibercept. ${ }^{17}$ In the current study, all eyes were treated with three injections of Ranibizumab followed by three injections of Aflibercept. The cases of refractory DME varied between the present study and Chen et al.'s, ${ }^{17}$ which likely explains the disparities in success rates. Rahimy et al. ${ }^{12}$ and Wood et al. ${ }^{18}$ also reported encouraging anatomical success rates. However, in both of these studies, patients were first given a combination of Bevacizumab and Ranibizumab before being switched to Aflibercept treatment. ${ }^{12,18}$ Laginhas et al. ${ }^{19}$ and Ashraf et al. ${ }^{20}$ reported that Aflibercept treatment reduced CMT and improved visual acuity in the eyes than the previous Bevacizumab treatment. In addition to refractory DME cases, promising results have been reported with Aflibercept treatment for macular edema in branch vein occlusion, ${ }^{21}$ central vein occlusion, ${ }^{22}$ and edema due to choroidal vasculopathy, ${ }^{23}$ which are frequent comorbidities of diabetic retinopathy or hypertension.

We found no significant improvement in vision after Aflibercept treatment. However, the anatomic success of intravitreal Aflibercept appears to be promising in many studies. The improvement of BCVA is debatable. For example, Chen et al. ${ }^{17}$ and Wood et al. ${ }^{18}$ reported significant improvements in vision, whereas Rahimy et al. ${ }^{12}$ did not report a significant improvement in BCVA. Variations in functional success definitions between studies may explain the differences in postAflibercept BCVA.

In the current study, higher CMT before switching anti-VEGF treatments was associated with lower success in reducing CMT and visual gain. Chen et al. ${ }^{17}$ reported a similar outcome. Thus, CMT may be a proxy indicator of longer duration of edema and non-response to Ranibizumab treatment regimen. Higher CMT prior to switching antiVEGF treatments could have irreversible damage to the foveal neuroreceptors, resulting in poor functional outcomes.

In the current study, the reduction in CMT after Aflibercept treatment was significantly higher in females than in males. In contrast, Chen et al. ${ }^{17}$ discovered no gender differences between responders and nonresponders to intravitreal Aflibercept. The higher prevalence of DR and sight-threatening diabetic retinopathy among males in the Saudi diabetic population suggests a greater need for anti-VGEF treatment in male diabetics. ${ }^{24}$ The different responses to Aflibercept based on gender reported in the current study warrant further investigation because it may influence strategies for reducing visual disabilities due to diabetes in the region. 
We discovered that age did not affect the efficacy of Aflibercept treatment. This observation is consistent with the findings of Chen et al. ${ }^{17}$ When compared to young type II diabetics, older patients with a longer duration of diabetes, advanced stages of DR, and reduced immunity are less likely to respond to anti-VEGF treatment. The issue of age and response to anti-VEGF therapy warrants further investigation better to understand the pharmacokinetics of Aflibercept treatment in DME.

Several protocols for switching steroids to different anti-VEGF compounds and combination therapy have been proposed. ${ }^{25,26}$ However, the introduction of intravitreal steroid implants has reduced the need for combination therapy or switching treatments for DME. Intravitreal steroid implants are effective and patient-friendly. Because they reduce the frequency of intravitreal injections. ${ }^{13}$ As a result, the cost-effectiveness and the anatomic and functional outcomes of anti-VEGF compounds must be evaluated to guide medical retina specialists in selecting the appropriate treatment regimen.

This study has some limitations. First, the retrospective single-arm cohort study design precludes comparison to a cohort that received a different treatment regimen.

In conclusion, intravitreal Aflibercept treatment appears to augment the benefits of intravitreal Ranibizumab in Saudi patients with DME. However, the visual improvements do not correspond with the antiVEGF treatment's high anatomical success. Because pretreatment CMT has an impact on final outcomes, switching should be done as soon as possible.

\section{CONFLICT OF INTEREST}

The authors declare that there is no conflict of interest.

\section{REFERENCES}

1. Diabetic Retinopathy Clinical Research Network, Wells JA, Glassman AR, Ayala AR, Jampol LM, Aiello LP, Antoszyk AN, et al. Aflibercept, bevacizumab, or ranibizumab for diabetic macular edema. N Engl J Med. 2015;372(13):1193-203. doi: 10.1056/NEJMoa1414264, PMID 25692915.

2. Bhagat N, Grigorian RA, Tutela A, Zarbin MA. Diabetic macular edema: Pathogenesis and treatment. Surv Ophthalmol. 2009;54(1):1-32. doi: 10.1016/j. survophthal.2008.10.001, PMID 19171208.

3. Blinder KJ, Dugel PU, Chen S, Jumper JM, Walt JG, Hollander DA, et al. AntiVEGF treatment of diabetic macular edema in clinical practice: Effectiveness and patterns of use (ECHO Study Report 1). Clin Ophthalmol. 2017;11:393-401. doi: 10.2147/OPTH.S128509, PMID 28260851.

4. Hussain RM, Ciulla TA. Treatment strategies for refractory diabetic macular edema: Switching anti-VEGF treatments, adopting corticosteroid-based treatments, and combination therapy. Expert Opin Biol Ther. 2016;16(3):365-74. doi: 10.1517/14712598.2016.1131265, PMID 26674182

5. Browning DJ, Stewart MW, Lee C. Diabetic macular edema: Evidence-based management. Indian J Ophthalmol. 2018;66(12):1736-50. doi: 10.4103/ijo. IJO_1240_18, PMID 30451174

6. Krick TW, Bressler NM. Recent clinically relevant highlights from the Diabetic Retinopathy Clinical Research Network. Curr Opin Ophthalmol. 2018;29(3):199-205. doi: 10.1097/ICU.0000000000000472, PMID 29528861.

7. American Academy of Ophthalmology. Diabetic retinopathy PPP; 2019 (in press) [cited 24/12/2019]. Available from: https://www.aao.org/preferredpractice-pattern/diabetic-retinopathy-ppp.

8. Abu El-Asrar AM. Evolving strategies in the management of diabetic retinopathy. Middle East Afr J Ophthalmol. 2013;20(4):273-82. doi: 10.4103/0974-9233. 119993, PMID 24339676.
9. Corcóstegui B, Durán S, González-Albarrán MO, Hernández C, Ruiz-Moreno JM Salvador J, et al. Update on diagnosis and treatment of diabetic retinopathy: A consensus guideline of the Working Group of Ocular Health (Spanish Society of Diabetes and Spanish Vitreous and Retina Society). J Ophthalmol. 2017;2017:1-10. doi: 10.1155/2017/8234186.

10. Khan AA, Rahmani AH, Aldebasi YH. Diabetic retinopathy: Recent updates on different biomarkers and some therapeutic agents. Curr Diabetes Rev. 2018;14(6):523-33. doi: 10.2174/1573399813666170915133253, PMID 28914194.

11. Do DV, Nguyen QD, Boyer D, Schmidt-Erfurth U, Brown DM, Vitti R, et al. da Vinci Study Group. One-year outcomes of the da Vinci Study of VEGF Trap-Eye in eyes with diabetic macular edema. Ophthalmology. 2012;119(8):1658-65. doi: 10.1016/j.ophtha.2012.02.010, PMID 22537617.

12. Rahimy E, Shahlaee A, Khan MA, Ying GS, Maguire JI, Ho AC, et al. Conversion to Aflibercept after prior anti-VEGF therapy for persistent diabetic macula edema. Am J Ophthalmol. 2016;164:118-27.e2. doi: 10.1016/j.ajo.2015.12.030, PMID 26748058.

13. Busch C, Zur D, Fraser-Bell S, Laíns I, Santos AR, Lupidi M, et al. International Retina Group. Shall we stay, or shall we switch? Continued anti-VEGF therapy versus early switch to dexamethasone implant in refractory diabetic macular edema. Acta diabetol. 2018:55(8):789-96. doi: 10.1007/s00592-018-1151-x, PMID 29730822.

14. Lim LS, Ng WY, Mathur R, Wong D, Wong EY, Yeo I, et al. Conversion to Aflibercept for diabetic macular edema unresponsive to ranibizumab or bevacizumab. Clin Ophthalmol. 2015;9:1715-8. doi: 10.2147/OPTH.S81523, PMID 26396494.

15. Al-Rashaed S, Alsulaiman SM, Alrushood AA, Almasaud J, Arevalo JF. Incidence of endophthalmitis after intravitreal anti-vascular endothelial growth factor: Experience in Saudi Arabia. Middle East Afr J Ophthalmol. 2016;23(1):60-3. doi: 10.4103/0974-9233.171756, PMID 26957840.

16. Majeed A, El-Sayed AA, Khoja T, Alshamsan R, Millett C, Rawaf S. Diabetes in the Middle-East and North Africa: An update. Diabetes Res Clin Pract. 2014;103(2):218-22. doi: 10.1016/j.diabres.2013.11.008, PMID 24300017.

17. Chen YY, Chang PY, Wang JK. Intravitreal aflibercept for patients with diabetic macular edema refractory to bevacizumab or ranibizumab: Analysis of response to aflibercept. Asia Pac J Ophthalmol (Phila). 2017;6(3):250-5. doi: 10.22608/ APO.2016186, PMID 28436640

18. Wood EH, Karth PA, Moshfeghi DM, Leng T. Short-term outcomes of aflibercept therapy for diabetic macular edema in patients with incomplete response to ranibizumab and/or bevacizumab. Ophthalmic Surg Lasers Imaging Retina. 2015;46(9):950-4. doi: 10.3928/23258160-20151008-08.

19. Laiginhas R, Silva MI, Rosas V, Penas S, Fernandes VA, Rocha-Sousa A, et al. Aflibercept in diabetic macular edema refractory to previous bevacizumab: Outcomes and predictors of success. Graefes Arch Clin Exp Ophthalmol. 2018 Jan 1;256(1):83-9. doi: 10.1007/s00417-017-3836-1, PMID 29082448.

20. Ashraf M, Kayal HE, Souka AAR. Safety and Efficacy of Ziv-Aflibercept in the Treatment of Refractory Diabetic Macular Edema. Ophthalmic Surg Lasers Imaging Retina. 2017;48(5):399-405. doi: 10.3928/23258160-20170428-06. PMID 28499051.

21. Konidaris V, Al-Hubeshy Z, Tsaousis KT, Gorgoli K, Banerjee S, Empeslidis T. Outcomes of switching treatment to aflibercept in patients with macular oedema secondary to central retinal vein occlusion refractory to ranibizumab. Int Ophthalmol. 2018;38(1):207-13. doi: 10.1007/s10792-017-0512-8, PMID 28405787

22. Konidaris VE, Tsaousis KT, Anzidei R, De la Mata G, Brent AJ. Real-world results of switching treatment from ranibizumab to Aflibercept in macular Oedema secondary to branch retinal vein occlusion. Ophthalmol Ther. 2018;7(2):387-95. doi: 10.1007/s40123-018-0149-3, PMID 30288685

23. Saito M, Kano M, Itagaki K, OguchiY, SekiryuT. Switching to intravitreal aflibercept injection for polypoidal choroidal vasculopathy refractory to ranibizumab. Retina. 2014;34(11):2192-201. doi: 10.1097/IAE.0000000000000236, PMID 25077530.

24. Yasir ZH, Hassan AD, Rajiv K. Diabetic retinopathy (DR) among 40 years and older Saudi population with diabetes in Riyadh governorate, Saudi Arabia - A population based survey. Saudi J Ophthalmol. 2019;33(4):363-8. doi: 10.1016/j. sjopt.2019.03.001, PMID 31920446

25. Treatment of diabetic macular edema. Am Acad Ophthalmol [website]

26. Mira F, Paulo M, Henriques F, Figueira J. Switch to Aflibercept in diabetic macular edema patients unresponsive to previous anti-VEGF therapy. J Ophthalmol. 2017;2017:5632634. doi: 10.1155/2017/5632634, PMID 28348885. 